\title{
PAESAGGI CULTURALI INTESI COME INTEGRAZIONE DI PROCESSI DINAMICI: IL VOMERO DA LUOGO DI DELIZIE A LUOGO URBANO
}

\section{CULTURAL LANDSCAPES AS AN INTEGRATION OF DYNAMIC PROCESSES: VOMERO FROM PLACE OF DELIGHTS TO URBAN PLACE}

MARIA MARTONE

Università Sapienza di Roma

\section{SINTESIS}

Il contributo illustra, attraverso gli strumenti della rappresentazione e del rilievo, le trasformazioni che il paesaggio della collina del Vomero a Napoli ha subito nel corso della storia, passando da "luogo di delizie" a "luogo urbano", continuando a caratterizzare il paesaggio dell'intera città. $\mathrm{A}$ tale scopo, si è ritenuto importante riconoscere e identificare, in uno spazio fisico, vissuto ed eterogeneo di un luogo, nel caso specifico, della collina del Vomero, i paesaggi culturali che contengono sia i segni del passato che quelli della contemporaneità. Delineare, quindi, quei processi dinamici che rappresentano la storia degli insediamenti umani in continua trasformazione, significa anche delineare il nostro patrimonio identitario, la cui conoscenza è necessaria per ritrovare il racconto di "chi siamo" e di "chi eravamo" e per costruire il futuro delle nuove generazioni.

Parole chiave: paesaggi culturali, territorio, Vomero, salvaguardia integrata, rilievo digitale. 


\section{ABSTRACT}

The contribution illustrates, through the tools of representation and relief, the transformations that the landscape of the hill of Vomero in Naples has undergone in the course of history, passing from "place of delights" to "urban place" continuing to characterize the landscape of the entire city. For this purpose, it was considered important to recognize and identify the cultural landscapes that contain both the signs of the past and those of contemporaneity, in a physical space, lived and heterogeneous of a place, in the specific case, the hill of Vomero. Outlining, therefore, those dynamic processes that represent the history of human settlements in continuous transformation, also means outlining our identity heritage, whose knowledge is necessary to find the story of "who we are" and "who we were" and to build the future of the new generations.

Keywords: : cultural landscapes, territory, Vomero, integrated preservation, digital relief.

Da ormai molti lustri si è configurato un nuovo concetto di paesaggio rivolto al territorio, i cui caratteri principali derivano "dalla natura, dalla storia umana e dalle reciproche interrelazioni", , contrapponendosi ad un pregresso giudizio estetico-percettivo, che considerava il paesaggio esclusivamente espressione di bellezze panoramiche o di quadri naturali ${ }^{2}$.

Vari aspetti concorrono in maniera integrata alla definizione del paesaggio come, ad esempio, la consistenza geografica, la realtà geologica, climatica, vegetazionale, ma anche e soprattutto l'attività economica, politica e culturale dell'uomo. Pertanto, la rappresentazione del paesaggio è riconducibile a due configurazioni fondamentali: quella naturale, che concerne i fattori biologici e fisiografici, e quella antropica, che concerne i fattori agroforestali ed insediativi ${ }^{3}$.

Il paesaggio può, quindi, definirsi espressione della sensibilità di un territorio $^{4}$, un territorio carico di identità e di valori culturali che si sono

1 Convenzione europea del paesaggio, Firenze 20 ottobre 2000, comma a, art. 1; Cf. Art. 131 del Decreto Legislativo 22 gennaio 2004, n. 42 “Codice dei beni culturali e del paesaggio, ai sensi dell'articolo 10 della legge 6 luglio 2002, n. 137'".

2 Cf. Legge n. 1089 del 1939: Tutela delle cose d'interesse artistico o storico; Legge n. 1497 del 1939 Protezione delle bellezze naturali. Successivamente la legge n. 1497 è stata integrata nel 1985 dalla legge n. 431 (denominata "legge Galasso") che ha esteso l'oggetto della tutela da cose singole o complessi di cose a intere porzioni di territorio, affermando la necessità di un governo del territorio attraverso l'istituzione del Piano Paesistico Regionale.

3 Cf. «Le categorie dei paesaggi», in Relazione al Piano Territoriale Paesistico Regionale, Lazio 2007.

4 MagnaGHI, A., Il principio territoriale, Torino, Bollati Boringhieri, 2020. 
consolidati e stratificati nel tempo ${ }^{5}$. Finalizzata alla conoscenza della qualità dei paesaggi e di tutto ciò che ha contribuito nel tempo a produrre paesaggio, è l'analisi dei processi di trasformazione, che si leggono nell'assetto paesistico di ogni luogo. Viene riconosciuta, pertanto, al paesaggio la sua natura dinamica come risultato del confronto dialettico tra un'istanza conservativa, a salvaguardia del passato, e le trasformazioni proprie della società. Una lettura e una documentazione critica del paesaggio non può che favorire il recupero della memoria di un rapporto che nel tempo l'uomo è riuscito a stabilire con il proprio territorio ${ }^{6}$. Il dato paesaggistico si presenta, insieme ed in stretto collegamento con quello archeologico, architettonico e letterario, come un fondamentale documento storico attraverso cui è possibile leggere in maniera unitaria le trasformazioni e le evoluzioni di un luogo. Possiamo, quindi, parlare di paesaggi culturali $^{7}$ che costituiscono il patrimonio identitario di un luogo.

Sulla base di tali presupposti e sulla base di una ricerca di recente pubblicata ${ }^{8}$, il contributo si propone di riconosce nel paesaggio attuale l'identità storica e culturale del Vomero, nonostante i numerosi interventi di edilizia speculativa lo abbiano in parte soffocato nel suo essere ambiente naturale e ambiente costruito. Con l'obiettivo di favorire una valorizzazione integrata territoriale, si è considerata la collina del Vomero come unico bene territoriale, con il suo sistema viario e edilizio, le ville e le masserie, in cui ogni singola architettura o piazza o strada arricchisce il contesto territoriale di cui ne fa parte, proponendo un modello integrato di indagine, basato su una documentazione multiscalare.

Il confronto e la sovrapposizione di spazi storici con gli spazi contemporanei hanno consentito l'identificazione delle permanenze attraverso cui si è potuto individuare il disegno delle trasformazioni che caratterizza il paesaggio contemporaneo nel suo aspetto dinamico. Attraverso, poi, il rilievo digitale di alcune architetture è stato possibile leggere il dettaglio architettonico come tassello importante ed essenziale che contribuisce alla costruzione dei paesaggi culturali.

Il Vomero da luogo di delizie, così come definito dal Celano nel 1692, quindi da spazio altro con carattere eterotopico rispetto al resto della città ${ }^{9}$ si trasforma 1993.

5 PURINI F., «Un paese senza paesaggio», in Il disegno del paesaggio italiano, Casabella, n. 575 -576,

6 MARTONE, M., «I paesaggi pontini: dalla macroscala all'elemento di dettaglio. Alcuni esempi», in CUNDARI, C., GIGLI, F. (a cura di). Paesaggi di città e territori. Roma, Aracne, 2011, p.100-116.

7 Il termine paesaggio culturale è stato per la prima volta usato dal geografo Otto Schluter nel 1908, termine che fu affiancato a paesaggio originario, ossia paesaggio esistente prima dei cambiamenti apportati dall'uomo.

8 Martone, M., La collina del Vomero. Da paesaggio agreste a forma urbana, Roma, Aracne Editrice, 2020.

9 FouCAUlt, M., Spazi altri. I luoghi delle eterotopie, a cura di VACCARO S., Udine, Mimesis Edizioni, 2011. 
in spazio pensato, progettato, pianificato, in uno spazio rappresentativo del potere di una istituzione, rappresentato nei disegni dei piani regolatori e di ampliamento di fine Ottocento, per trasformarsi ancora in spazio vissuto ${ }^{10}$, così come viene rappresentato nei disegni delle scene urbane delle Riviste illustrate nazionali ed europee dei primi anni del Novecento ${ }^{11}$.

È proprio lo spazio vissuto, che contiene sia lo spazio reale che quello pensato, sia lo spazio materiale che immateriale, sia quello percepito che quello concepito, che ci conduce alla definizione di uno terzo spazio ${ }^{12}$ ossia di uno spazio ibrido, da cui partire per il riconoscimento dei paesaggi culturali.

\section{IL VOMERO COME "LUOGO DI DELIZIE"}

L'iconografia storica di Napoli fino alla fine dell'Ottocento ha riprodotto l'immagine di una città di mare circondata dalle verdi colline di Posillipo, Camaldoli, Vomero e Capodimonte, in cui sorgevano accanto a casolari, ville e piccoli nuclei agresti, architetture monumentali come Castel Sant'Elmo con la Certosa, il palazzo di Capodimonte, l'eremo dei Camaldoli. La città si estendeva in direzione del mare fino al monte Echia ${ }^{13}$, fermandosi ad est dove il territorio si presentava paludoso ${ }^{14}$ (Fig. 1).

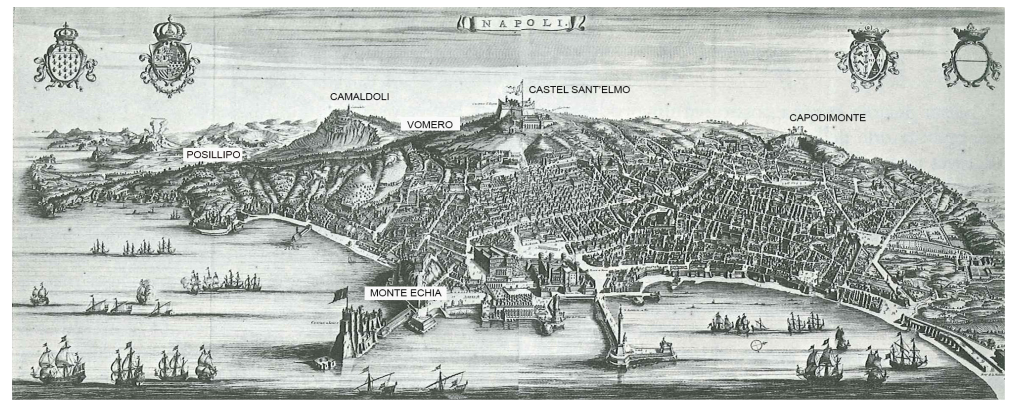

Fig. 1. Napoli, Basiaen Stopendaal, Amsterdam 1704 (Pane, Valerio, 1987, p. 196). In evidenza: Castel Sant'Elmo, il monte Echia e le colline di Posillipo, Camaldoli, Vomero e Capodimonte.

10 LeFEBVRE, H., La produzione dello spazio, Milano, Pgreco Edizioni, 2018.

11 Pallottino, P., Storia dell'illustrazione italiana, Bologna, Zanichelli, 1988; Giordano, M., La stampa illustrata in Italia 1834-1915, Milano, Guanda, 1983.

12 SoJA, E., Thirdspace: Journeys to Los Angeles and Other Real and Imagined Places. Cambridge (USA), Blackwell, 1996.

13 Sul monte Echia sorse il primo nucleo urbano denominato Partenope.

14 Summonte, A., Historia della città e Regno di Napoli, Napoli, Stamperia di Domenico Vivenzio, 1748, Tomo primo dedicato all'eccellentissima città di Napoli, p. 305-318. 
Le colline di Napoli costituivano dei veri e propri luoghi di delizie ${ }^{15}$, luoghi ameni, caratterizzati da un'aria salubre. Accanto a stradine strette, ripide, tortuose, che collegavano la collina alla città di pianura, il Vomero era attraversato, fin dall'epoca romana, da una importante arteria stradale la via per colles, che collegava Pozzuoli a Napoli ${ }^{16}$ (Fig. 2).

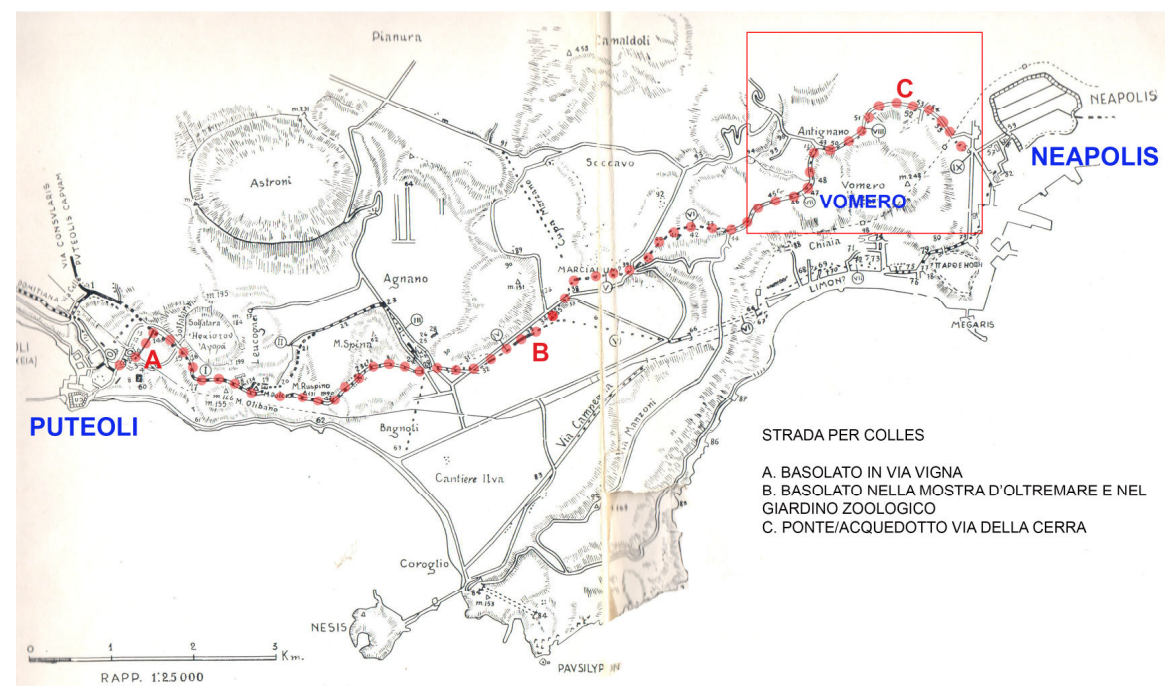

Fig. 2. Mappa del litorale tra Pozzuoli e Napoli (Werner, 1952). In evidenza il percorso della strada romana "per colles" con l'ubicazione di alcuni reperti archeologici e la zona del Vomero.

Immettendosi lungo la direttrice dei collegamenti Roma-Napoli, la strada è stata fondamentale per lo sviluppo del Vomero, rappresentandone la viabilità principale, da cui si diramavano numerosi percorsi secondari.

Lungo il suo tracciato sono sorti i casali del Vomero, Antignano e Arenella con ville e masserie, che con il tempo si sono ampliati a macchia d'olio.

Tracce dell'antico basolato della strada romana sono state ritrovate in prossimità di Pozzuoli in via Vigna e nella conca di Agnano, presso Fuorigrotta,

15 CELANO, C., Notizie del bello, dell'antico e del curioso della città di Napoli, Napoli, 1692, nella Edizione a cura di CHIARINI, G. B., Napoli, Stamperia di Nicola Mencia, 1859, vol. IV, p. 745-753. Giornata Sesta.

16 Werner J., Contributi alla Campania antica. La via Puteolis Neapolim, Napoli, Arte Tipografica, 1953. Su questo argomento l'autore sta svolgendo approfondimenti all'interno del Progetto di Ricerca di Interesse Nazionale - Prin 2017 "Stone paviments. History, conservation, valorisation and design" (20174JW7ZL) finanziato dal Ministero dell’ Istruzione, Università e Ricerca (MIUR). 
all'interno della Mostra d'Oltremare ${ }^{17}$ e nel giardino zoologico di Napoli, mentre lungo il tratto collinare, indicato successivamente nella cartografia storica con il nome di Infrascata, è stata rinvenuta, una struttura muraria con archi di epoca romana. Si tratta di resti rinvenuti durante i lavori della costruzione della stazione della Linea 1 della metropolitana, appartenenti o ad un viadotto della antica strada romana o ad un acquedotto che serviva la zona flegrea che caratterizzano oggi il paesaggio in modo molto significativo insieme alle opere architettoniche ed artistiche della stazione d'arte di Salvator Rosa $^{18}$ (Fig. 3). La via per colles, con le sue diverse denominazioni, divenne l'arteria principale del sistema viario collinare, spingendosi anche verso l'interno per raggiungere zone una volta inabitate ed incolte che si estendevano sulla superfice del vasto altopiano collinare del Vomero.

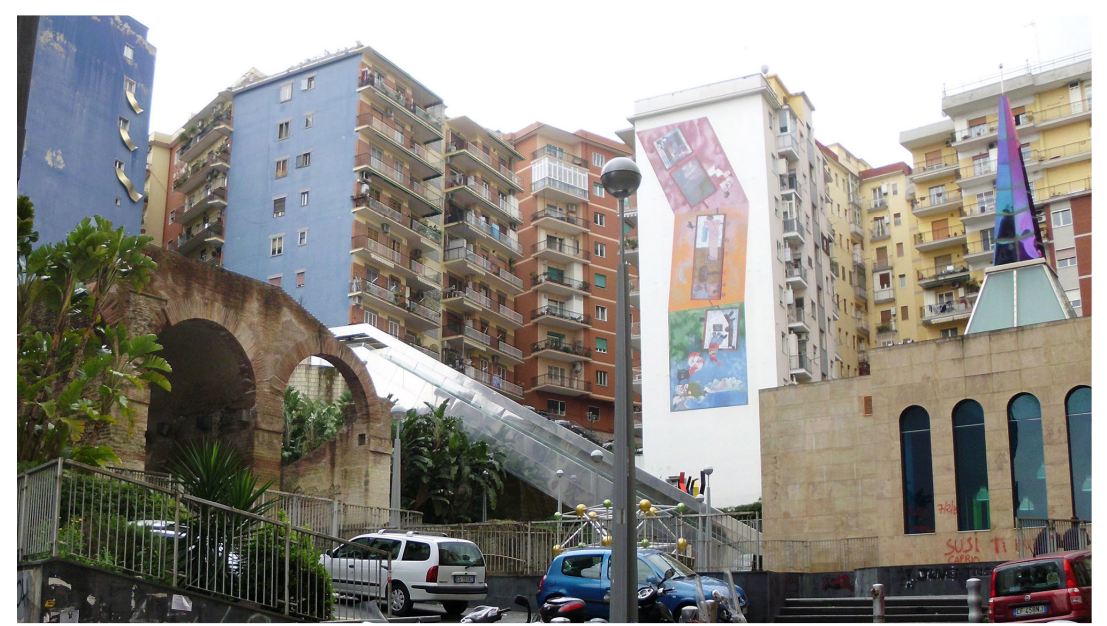

Fig. 3. Via Conte della Cerra, Vomero. In primo piano sulla sinistra i ruderi di un antico ponte/acquedotto rinvenuto lungo il percorso della strada per colles. Foto dell'autore.

In prossimità di S. Stefano, all'ingresso del Vomero, l'antica strada romana prese il nome di: strada che porta al Vomero, per poi chiamarsi strada dell'Infrascata, così come si evince dalla "Mappa topografica della città di

17 MARTONE, M., «The Roman road "per colles" between Puteoli and Neapolis. The drawing of some testimonies», in GAmBARDELla, C., World Heritage and Design for Health, Le vie dei Mercanti, Roma, Gangemi Editore International, 2021, p. 92-101.

18 MARTONE, M., PAPA, F., «Colori, luci e forme nell'underground napoletano. Le "stazioni dell'arte" della Linea 1 - Colors, lights and shapes in the Naples underground. The "art stations" of Line 1», in Cultura e Scienza del Colore - Color Culture and Science, n.10/2018, Milano, Gruppo Del Colore Associazione Italiana Colore-editrice della rivista in formato elettronico, 2018, pp. 105-120. 
Napoli e de' suoi contorni" di Giovanni Carafa, duca di Noja del $1750^{19}$. La strada presentava due diramazioni in prossimità del casale detto il Vomero, che si spingevano fino al Castel Sant'Elmo, mentre in prossimità del casale Antignano si sviluppava un ramo verso l'Arenella ed un altro verso ovest. L'antico sistema viario è stato identificato nel tessuto attuale del Vomero attraverso la Carta Tecnica Regionale della Campania del comune di Napoli.
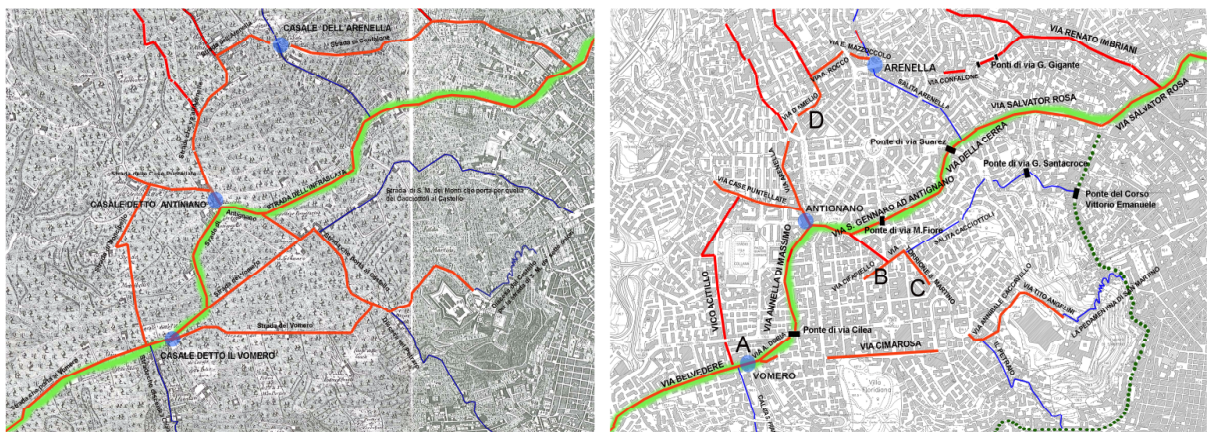

Fig. 4. A sinistra: Mappa Topografica della città di Napoli e de' suoi contorni (fogli 10 e 11), di G. Carafa duca di Noja, incisione su rame, 1775 (Pane, Valerio, 1987, pp. 281, 282). In evidenza il sistema stradale di collegamento tra i Casali sulla collina del

Vomero con la relativa toponomastica. In verde la via per colles, in rosso le diramazioni principali e in blu le secondarie. A destra: Planimetria del quartiere Vomero-Arenella tratta dalla Carta Tecnica Regionale (C.T.R.) della Campania del comune di Napoli, fogli 447112 e 447152, scala originaria 1: 5.000

(https://sit2.regione.campania.it/content/carta-tecnica-regionale-2004-2005). In evidenza: l'antico tracciato viario ancora presente; l'ubicazione dei ponti per la realizzazione dell'impianto stradale otto-novecentesco. Le lettere da A a D fanno riferimento a quanto illustrato nella figura 5.

In tal modo, si è potuto, constatare che, pur essendo in parte ancora presente ed utilizzate nella viabilità del Vomero ${ }^{20}$, le antiche strade hanno perso, in seguito alle trasformazioni urbane della fine Ottocento e a quelle ancora successive, il valore territoriale di un tempo (Fig. 4). Forte è il contrasto con l'impianto viario ottocentesco realizzato con la costruzione del nuovo quartiere, in cui però l'antico percorso esprime ancora una valenza storica significativa

19 La pianta, conclusasi nel 1775 dopo sette anni la sua morte e redatta in 35 tavole in scala 1:3080 restituisce il territorio della città di Napoli e dei suoi sobborghi così come si presentava nel XVIII secolo. Realizzata sulla base di un rilievo topografico eseguito adottando la tavoletta pretoriana, la mappa rappresenta il rilievo dell'intera superficie urbana e l'estensione del territorio rappresentato fa apparire il rilievo più una mappa territoriale che una pianta urbana. Cf. PANE, R., VALERIO V., a cura di, La città di Napoli tra vedutismo e cartografia. Piante e vedute dal XV al XIX secolo., Grimaldi \& e C. Editori, Napoli 1987.

20 La strada per colles ha preso, successivamente, il nome di via Belvedere, via Gino Doria, via Annella di Massimo, via S. Gennaro ad Antignano, via Conte della Cerra e via Salvator Rosa. 
che si legge nelle architetture superstiti di un paesaggio storico ancora presente, ma che purtroppo esprime una consistente fragilità (Fig. 5).
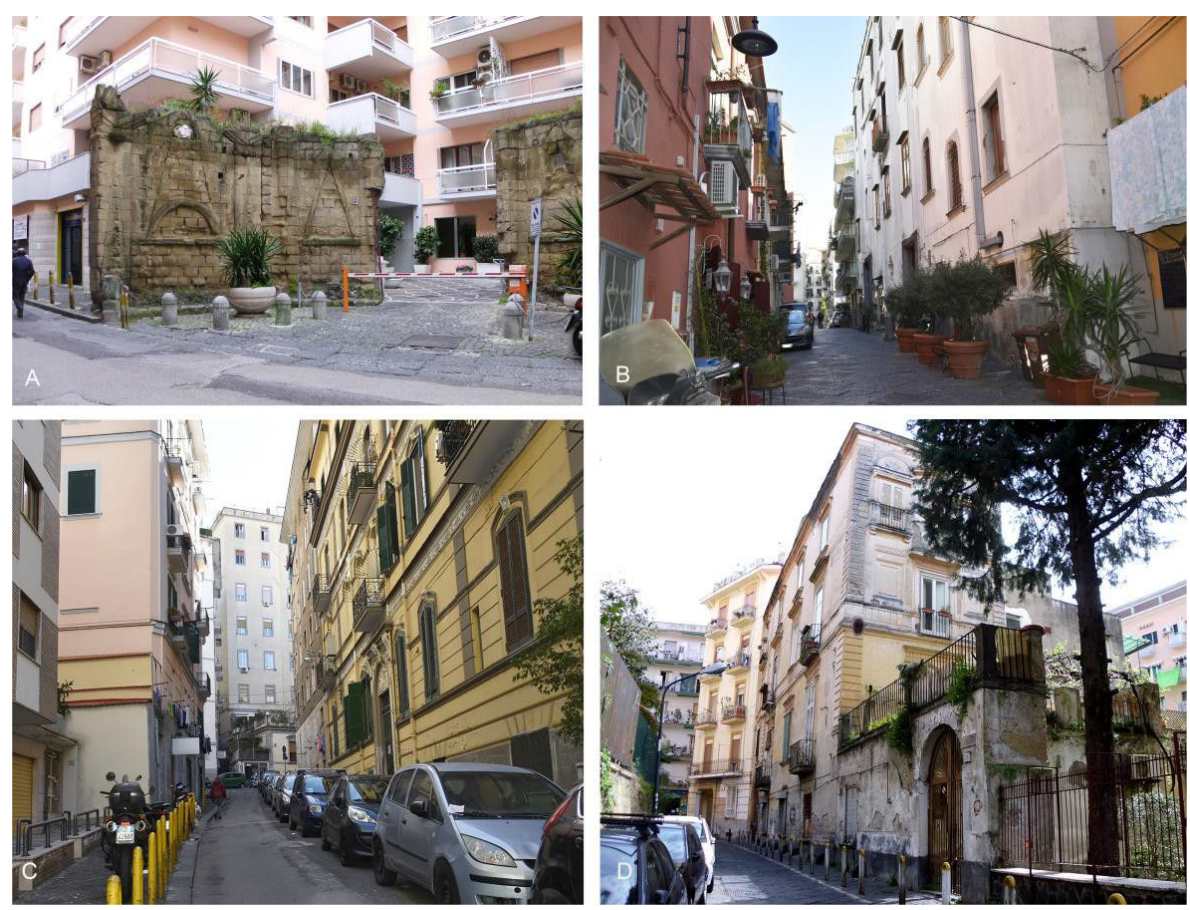

Fig. 5. Vedute di alcuni tratti dell'antico impianto viario sopravvissuto al progetto di ampliamento della fine Ottocento: A) esedra in tufo davanti a villa Belvedere nella via omonima, tagliata per consentire l'ingresso al condominio retrostante; B) via Cifariello; C) via Torrione S. Martino; D) villa Cantone in via D'Amelio. Foto dell'autore.

La funzione di luogo di riposo, di svago e di villeggiatura, di "spazio altro" che il sistema collinare del Vomero iniziò ad assumere nel XV secolo, si rafforzò soprattutto nelle epoche successive quando accanto alle antiche masserie contadine si affiancarono ville residenziali di nobili che sulle pendici della collina costruirono le loro seconde case. La definizione di "spazio altro"21 ben rappresenta il Vomero fino all'inizio Ottocento, in cui il rapporto con l'ambiente naturale rispetto alla città bassa, caotica e densamente popolata, continua a caratterizzare il modo di vivere degli abitanti in collina. 

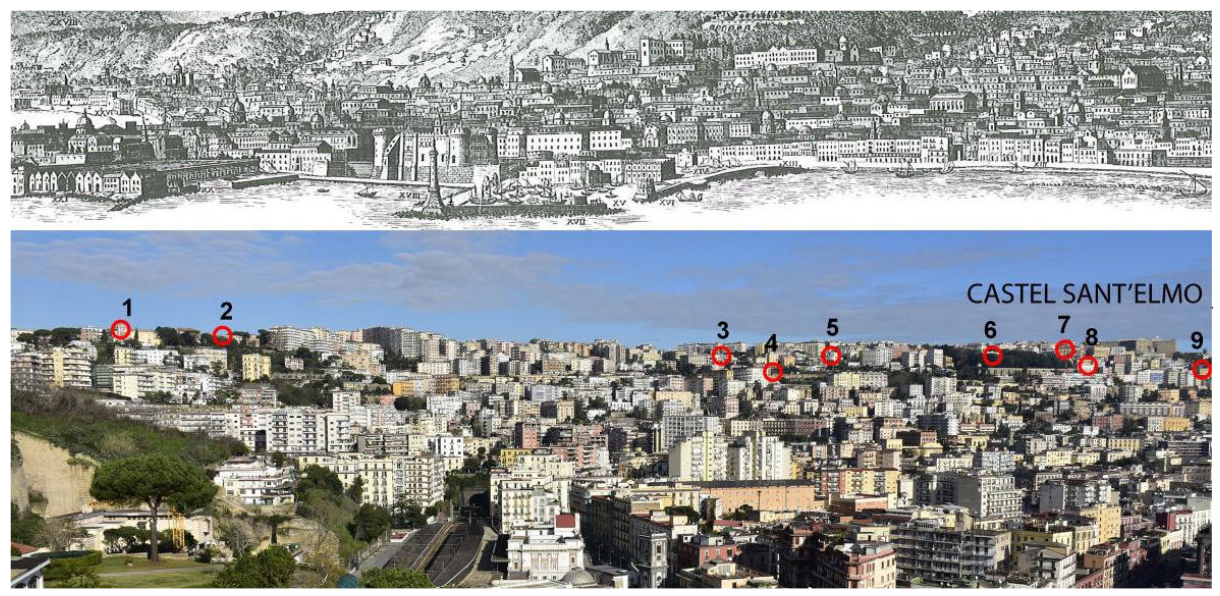

Fig. 6. In alto: vista di Napoli a Ponente di G. Carafa duca di Noja, 1775, stralcio (Pane, Valerio, 1987, p. 297). In evidenza le ville: Belvedere (1), Pietracatella (2), Ruffo-Palazzolo-Haas (3) e Giannone (4). In basso: veduta fotografica attuale di Napoli realizzata dall'autore dal piazzale antistante la chiesa di S. Antonio a Posillipo. Sono state individuate le ville: Salve (1); Ricciardi (2); Giordano (3), Belvedere (4), Buonocore (5); Floridiana (6), Pietracatella (7), Ruffo-Palazzolo-Haas (8) e De Rosa (9).

Accanto alle ville suburbane sorsero anche numerosi complessi religiosi, tra cui ricordiamo Santa Maria della Libera del XVI secolo in via Belvedereattorno al quale si sviluppò il primo nucleo abitato del Vomero-e Santa Maria del Soccorso all'Arenella del XVII secolo, che rappresentò la prima parrocchia collinare.

La via per colles, con le sue diverse denominazioni a differenziare i luoghi che attraversava, divenne l'arteria principale del sistema viario collinare, spingendosi anche verso l'interno per raggiungere zone una volta inabitate ed incolte che si estendevano sulla superfice del vasto altopiano collinare del Vomero.

Le ville e le case nobiliari costruite lungo il crinale o ad esso sottostante erano visibili dal mare, da Posillipo, da Pizzofalcone e da San Martino. Numerose sono state, infatti, le vedute panoramiche realizzate fino a tutto il XIX secolo della città di Napoli sia dipinte che incise, da cui si può individuare il profilo della collina del Vomero con Castel Sant'Elmo. È nel Settecento, con l'inizio del vedutismo, che si arricchisce il patrimonio iconografico della città di Napoli. Da diversi punti di vista viene restituita l'immagine di un ambiente urbano in stretto rapporto con il contesto naturale. Una veduta particolare ed inconsueta in quanto 
ritrae la città di Napoli a $360^{\circ}$ è quella di Georg Friedrich Heilmann de Rondchatel eseguita nel 1841 dal piazzale di Castel Sant'Elmo ${ }^{22}$ (Fig.7).

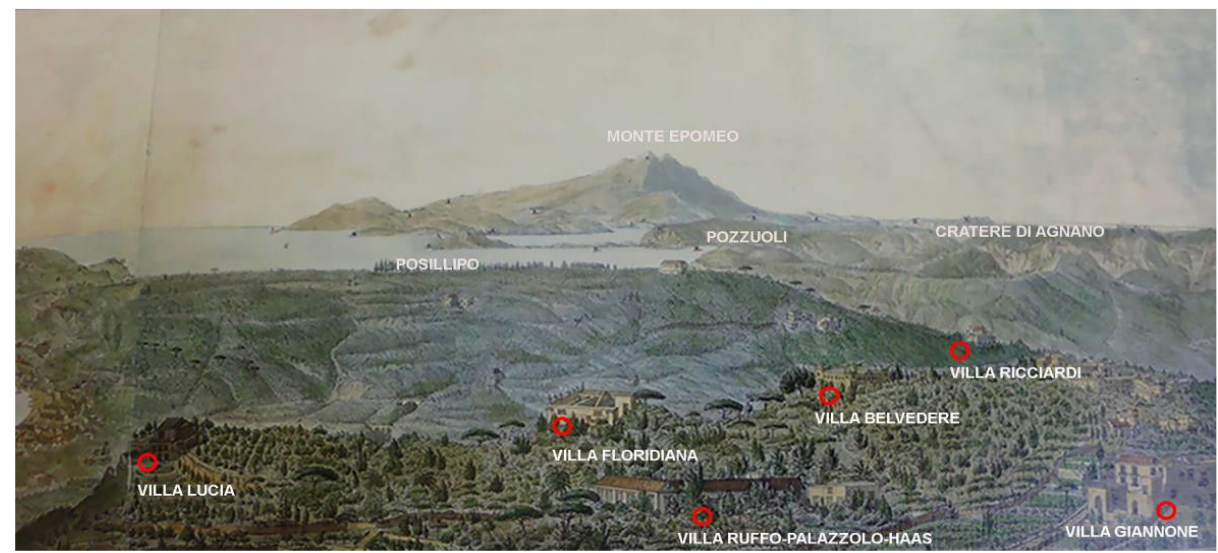

Fig. 7. Il panorama di Napoli e i suoi dintorni di G. F. Heilmann de Rondchatel, 1849.

Tavola VI. (Zampino, 1990). In evidenza i dintorni di Napoli e alcune ville riconoscibili tra il verde della collina del Vomero.

L'aspetto che il Vomero ha avuto dai primi insediamenti in epoca romana fino alla fine dell'Ottocento, confrontato con il resto della città sottostante caotica e con una alta densità abitativa, si basa su un concetto di eterotopia, in quanto esprime qualcosa di altro separato dalla norma, qualcosa che ha consentito di vivere orizzonti diversi da quelli che la realtà poteva all'epoca offrire nella città bassa. Si può constatare, quindi, come particolari siano stati gli ambienti naturali e artificiali che hanno dato vita al Vomero come luogo di delizie, di cui oggi possiamo a fatica ritrovare solo pochi elementi superstiti, come tratti di strada, di ville, di masserie, di decorazioni architettoniche.

Tuttavia, sono proprio questi elementi che, sommandosi al palinsesto visivo e informativo che la rete ci offre anche in tempo reale, rappresentano gli elementi essenziali, $i$ tasselli fondamentali per la lettura dei paesaggi culturali a testimonianza di una realtà che si è trasformata.

Il vomero come "luogo urbano" nel progetto di nuova espansione tra il XIX e il XX secolo

22 L'artista utilizza un prospettografo, in sostituzione della camera oscura, consistente in un telaio quadrettato in cui veniva inquadrata la scena da rappresentare, e girando progressivamente la strumentazione disegnò sei vedute contigue riprendendo l'intera scena paesistica ed urbana, avendo come riferimento l'Eremo dei Camaldoli. Il Vomero è rappresentato nella sesta veduta. Cf. ZAMPINO, G., a cura di, Il panorama di Napoli e $i$ suoi dintorni, disegnato dal Castel S. Elmo da G. F. Heilmann de Rondchatel, Napoli, Grimaldi \& e C. Editori, 1990. 
L'aspetto bucolico e agreste della collina del Vomero, che si legge nelle vedute e nella cartografia antica dei secoli XVIII e XIX, si trasformò in seguito al piano di risanamento dei quartieri bassi e di ampliamento della città di Napoli del 1885 a favore di una urbanizzazione dei terreni che, cambiando la destinazione d'uso da agricola a residenziale, furono aggregati alla città di pianura. Una maglia uniforme di strade parallele e radiali si sovrappose ai terreni della collina, dando vita nell'altopiano ad un nuovo quartiere che, in contrapposizione ai villaggi preesistenti, proponeva una edilizia a cortina basata sull'uso intensivo del lotto (Fig. 8).

Il progetto del 1885, a cura di Adolfo Giambarba, in adesione ai concetti teorici ed operativi definiti in Europa nei programmi di espansione e di miglioramento delle condizioni igienico-sanitarie delle città, prevedeva per il centro della città di Napoli il diradamento dell'edilizia esistente attraverso la demolizione di fondaci e di vicoli e la ricostruzione per "isolati" del tessuto edilizio con una nuova rete stradale, mentre le zone periferiche sia in pianura che in collina, tra cui anche il Vomero, furono coinvolte dal piano di ampliamento della città ${ }^{23}$.

Il concetto informatore del progetto di ampliamento, che investì anche la collina del Vomero, consisteva nel realizzare strade il più possibile dritte ${ }^{24}$, alternate con un sistema di piazze e formanti reticoli squadrati e radiali, con isolati di regolari dimensioni, nella logica dei modelli accademici, mostrando poca sensibilità ai problemi paesistici e all'orografia del luogo, privilegiando una edilizia a cortina secondo le tipologie codificate dell'epoca. Le nuove strade della collina del Vomero si sarebbero poi sovrapposte a quelle preesistenti, prevedendo anche la demolizione di alcuni edifici e antichi tracciati viari. Rimase, comunque, precario il collegamento con il centro, nonostante le realizzazioni delle due funicolari di Chiaia e di Montesanto ${ }^{25}$, già previste prima della costruzione del quartiere, e della linea tranviaria che collegava piazza Vanvitelli con piazza Dante. Il disegno di progetto del nuovo

23 In pianura sorse un quartiere ad oriente della città, nella zona paludosa di Vasto bonificata attraverso lavori di colmata, includendo case economiche, destinate agli abitanti delle case demolite. Inoltre, fu previsto anche un ampliamento ad est presso la Riviera di Chiaia con il rione Amedeo. I quartieri progettati in collina sorsero, invece, a Capodimonte e nella zona dei Casali Vomero-Arenella-Antignano. Cf. Russo, G., Il Risanamento e l'Ampliamento della città di Napoli, Napoli, Società pel Risanamento, 1959.

24 COCCHIA, C., L'edilizia a Napoli tra il 1918 e il 1958, Napoli, Società pel Risanamento di Napoli, 1961, p. 112. La gara di appalto fu vinta dalla Società pel Risanamento di Napoli che si impegnò a portare a termine entro dieci anni i lavori previsti, scadenza che non fu rispettata per le particolari condizioni economiche in cui versavano le banche in quel periodo.

25 MARTONE M, «I collegamenti su fune dalla collina del Vomero al centro della città di Napoli tra il XIX e il XX secolo», in: Norba, Revista de Arte, Vol. 34/2014. Universidad de Extremadura, Caceres 2015, p. 105121. 
quartiere presentava un impianto stradale a maglie ortogonali avente come assi principali le attuali vie Alessandro Scarlatti e Gian Lorenzo Bernini nella cui intersezione fu prevista una piazza poi denominata piazza Vanvitelli.

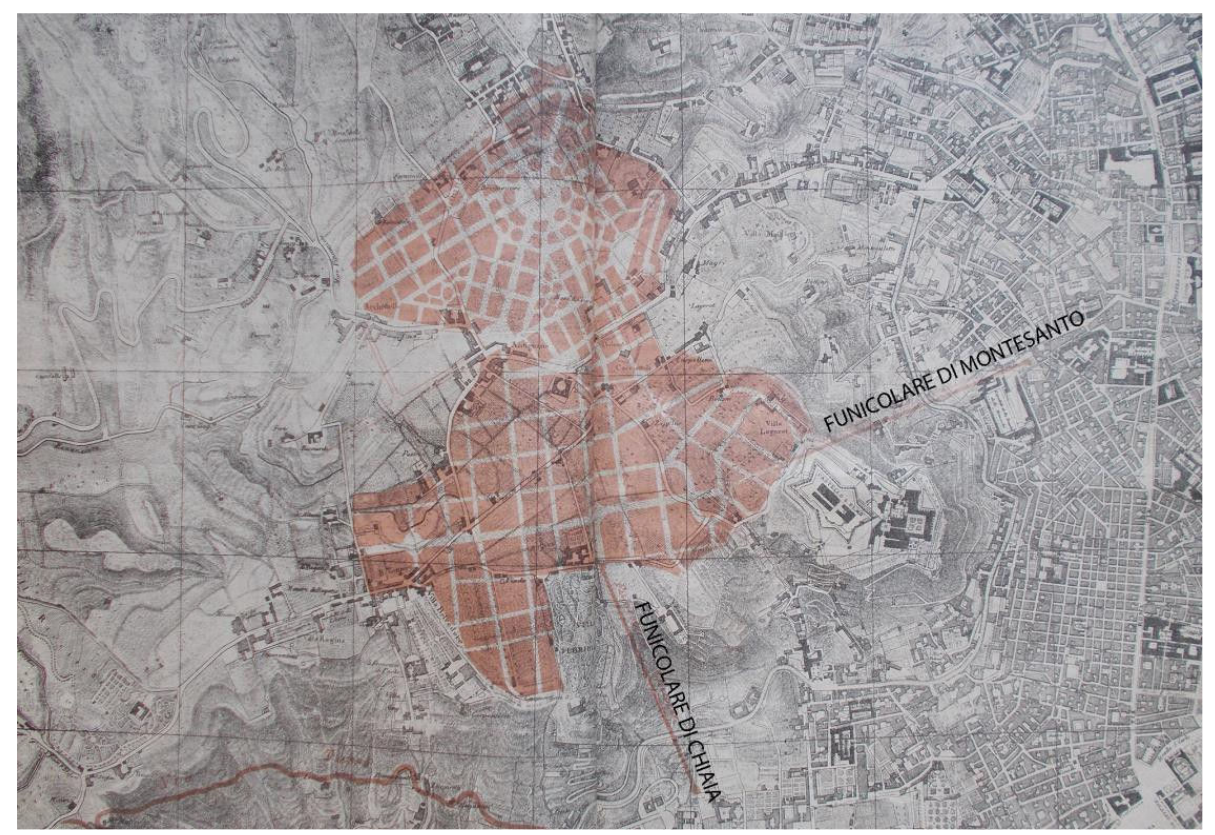

Fig. 8. Pianta dei nuovi rioni Vomero e Arenella, Bollettino Ingegneri Architetti, n. $2-3,1886$, pp. $12-13$. Prima stesura. In evidenza le funicolari di Chiaia e di Montesanto.

Parallelamente a via Bernini, nella direzione nord-sud, fu progettata via Luca Giordano, che si fermava ad Antignano e solo successivamente il suo tratto finale fu deviato verso est, per evitare la demolizione della casa di Giovanni Pontano ${ }^{26}$. L'asse perpendicolare a via Scarlatti, via Bernini, confluiva direttamente in una grande piazza ottagonale l'attuale piazza delle Medaglie d'Oro, per raggiungere, sempre in rettilineo, il casale dell'Arenella, nell'attuale piazza Francesco Muzii. Dalla piazza ottagonale partivano a raggiera otto strade, una delle quali Tino di Camaino raggiungeva l'antico casale di Antignano, nell'attuale piazza degli Artisti (Fig. 9).

26 Alisio, G., Il Vomero, Napoli, Electa, 1987. Il tratto superiore di via Scarlatti, ad est, fu progettato con due tornanti (l'attuale via Raffaele Morghen) e con una scalinata, attraverso cui poter raggiungere castel Sant'Elmo e la funicolare di Montesanto. Nel tratto inferiore, ad ovest, via Scarlatti proseguiva in rettilineo nella zona Belvedere con l'attuale via Francesco Cilea. 


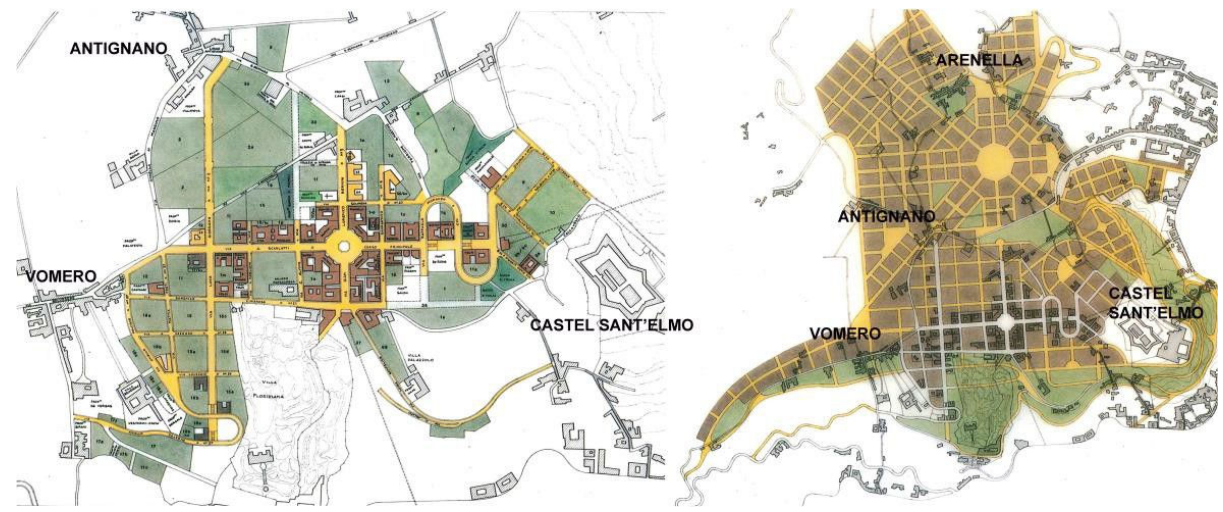

Fig. 9. A sinistra lo stato dei luoghi del progetto del nuovo quartiere al 1889 a destra il piano regolatore del 1914 redatto dall'ing. F. De Simone. In entrambe le planimetrie sono stati evidenziati i casali Vomero, Arenella e Antignano, e Castel Sant'Elmo (Alisio, 1987).
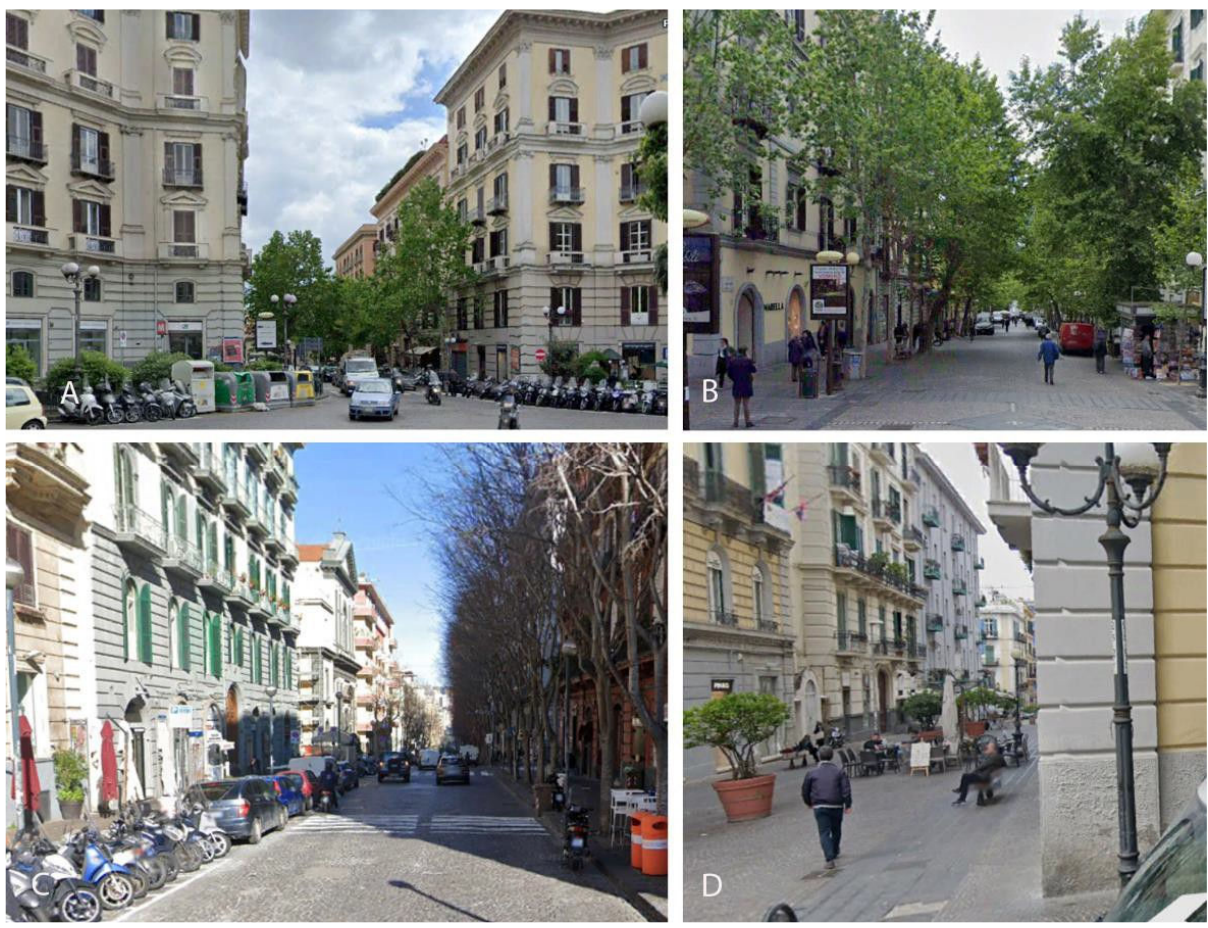

Fig. 10. Vedute su: piazza Vanvitelli (A), via Scarlatti (B), via Bernini (C), e in via Merliani (D). Foto da Google Earth Pro. 
Si trattava di «lunghe strade rettilinee di sezione molto ampia, per solito alberate alla moda del tempo, coordinate a piazze secondo un andamento assiale ${ }^{27}$ (Fig. 10).

I collegamenti all'interno del nuovo rione Vomero Arenella furono incrementati con il servizio degli omnibus, diligenze a cavallo destinate al trasporto pubblico.

Le opere iniziarono ufficialmente il 15 giugno 1889 e diverse furono le leggi che si susseguirono per l'attuazione del piano.

L'impianto ottocentesco del quartiere con le sue imponenti cortine degli isolati ha rappresentato una chiusura verso l'ambiente esterno e soprattutto verso un paesaggio che, per la sua unicità, non avrebbe dovuto trovare ostacoli alla sua percezione soprattutto nei punti più significativi, come ad esempio, in prossimità del crinale della collina. «Non un disegno diverso del quartiere, bensì una intensità edilizia assai più rada e tipologie differenti sarebbero state auspicabili, ma questo sarebbe stato in contrasto con l'idea capitalistica e ottocentesca della città ${ }^{28}$ (Fig. 11).
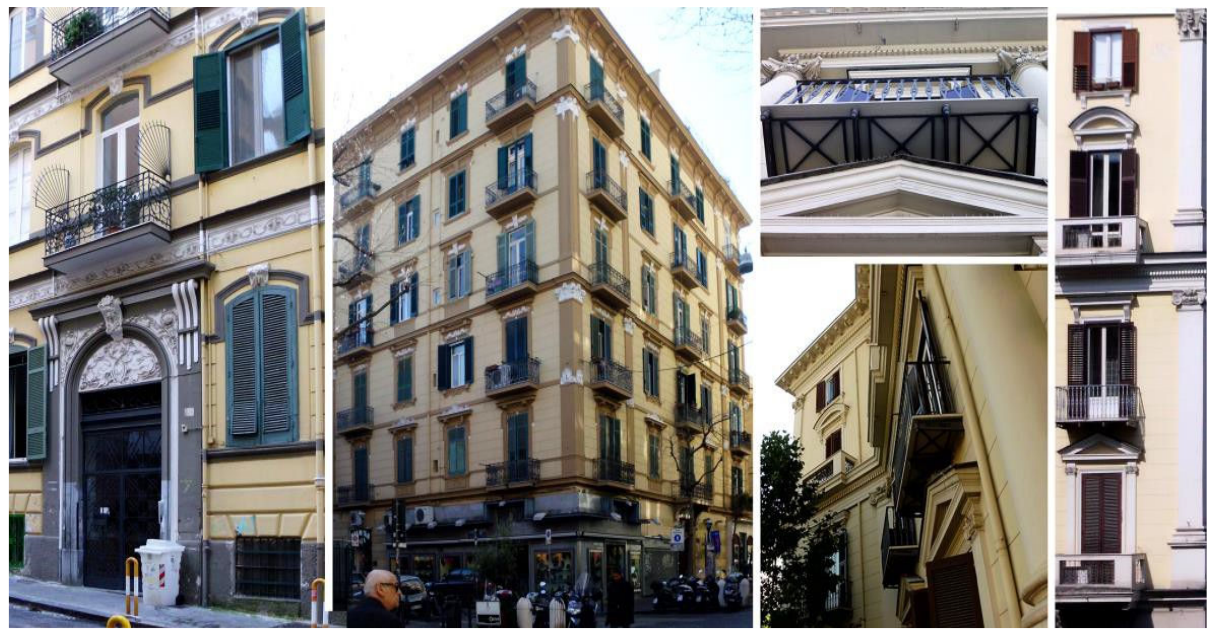

Fig. 11. Palazzi neoclassici in: via Torrione S. Martino, angolo tra via Scarlatti e via Luca Giordano. Dettagli architettonici dell'edificio in piazza

Vanvitelli 24. Foto dell'autore. 


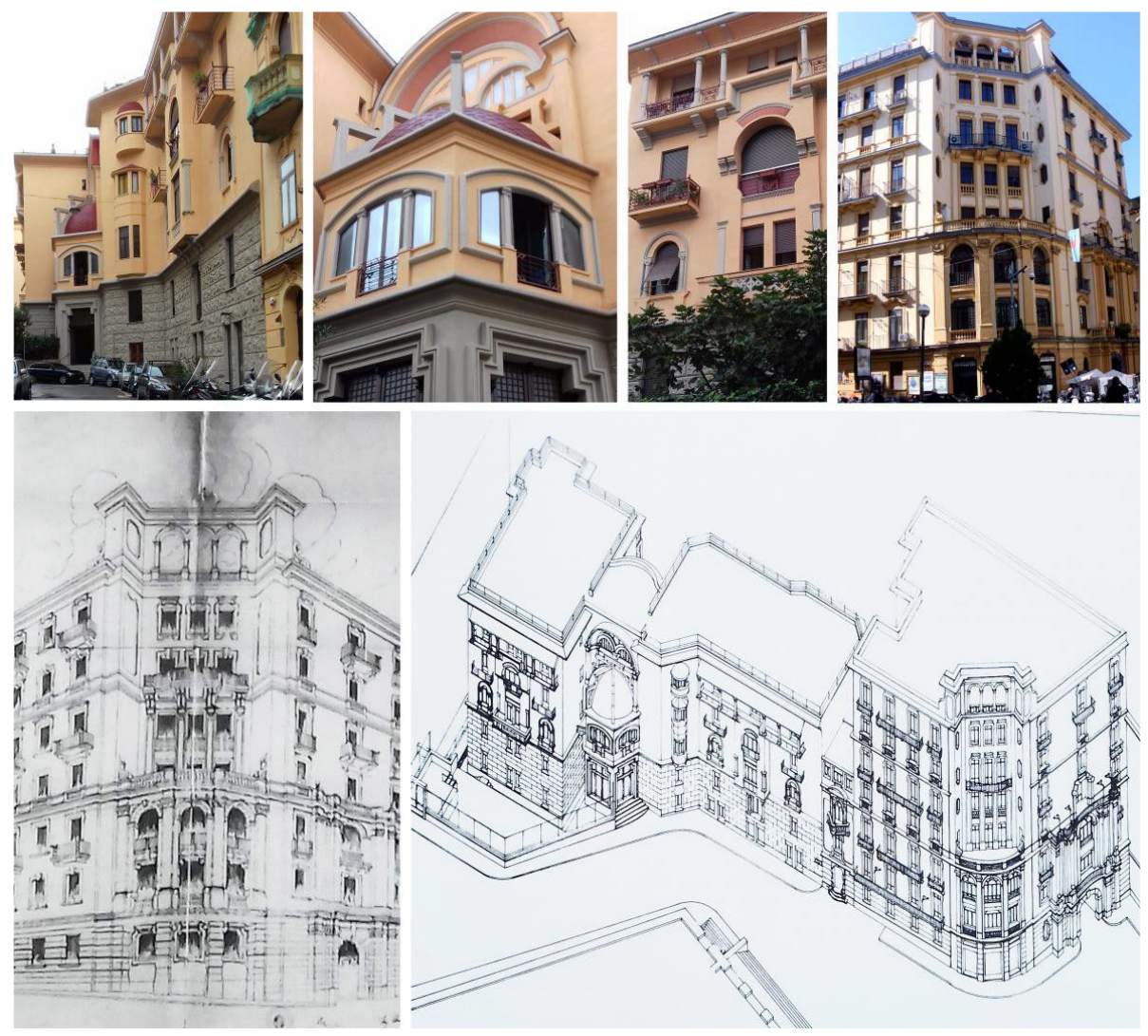

Fig. 12. Palazzina in via Lordi 6 di Adolfo Avena. In alto: soluzioni architettoniche delle facciate (foto dell'autore). In basso: Disegno di Adolfo Avena dal progetto (De Seta, 1999), assonometria dell'edificio (Gambardella, De Falco, 1991).

La collina del Vomero trovava il suo punto di maggiore pendenza in prossimità del colle di S. Martino dove si innalza Castel Sant'Elmo e, mentre verso est degradava lentamente, ad ovest le sue pendici risultavano alquanto ripide verso via Conte della Cerra e via Salvator Rosa ${ }^{29}$. Agli imponenti fabbricati di stile neorinascimentale si affiancò nel primo ventennio del XX una edilizia realizzata per piccoli lotti con edifici di due o tre piani con caratteristiche architettoniche protese all'affermazione di un nuovo gusto

29 La banca che investì capitali per la costruzione del nuovo quartiere fu la Banca Tiberina, a cui subentrò successivamente la Banca d'Italia in seguito a numerose difficoltà economiche. Per facilitare gli investimenti privati si adottò il processo di frazionamento dei lotti, per una vendita più veloce e conveniente. All' interno dello schema a scacchiera, che prevedeva isolati regolari, si sviluppò una edilizia, caratterizzata da una tipologia a cortile chiuso o a forma di " $\mathrm{C}$ " con all'interno un cortile/giardino, di cui permangono ancora molti esempi lungo le strade principali. 
floreale ed eclettico ${ }^{30}$ tra cui ricordiamo la palazzina in via Lordi 6 di Adolfo Avena $^{31}$ (Fig. 12). Molte di queste fabbriche sono state purtroppo demolite e sostituite con interventi di edilizia speculativa che hanno notevolmente modificato l'immagine del primo quartiere ${ }^{32}$.

Solo dopo alcuni decenni si realizzò il nuovo rione Arenella ${ }^{33}$ in continuità con il rione Vomero, ma ancora da esso separato dal vallone dell'Infrascata. L'impianto urbanistico risultò, infatti, molto rigido per un territorio che presentava una orografia molto varia e caratterizzata da notevoli salti di quota e vari dislivelli.

L'orografia dei luoghi ha, infatti, condizionato l'impianto del quartiere anche se attualmente da un primo impatto con la zona ciò non si percepisce, perché tutto ormai è nascosto da una stratificazione edilizia che nel tempo è diventata sempre più densa, facendo perdere il contatto con la morfologia del suolo.
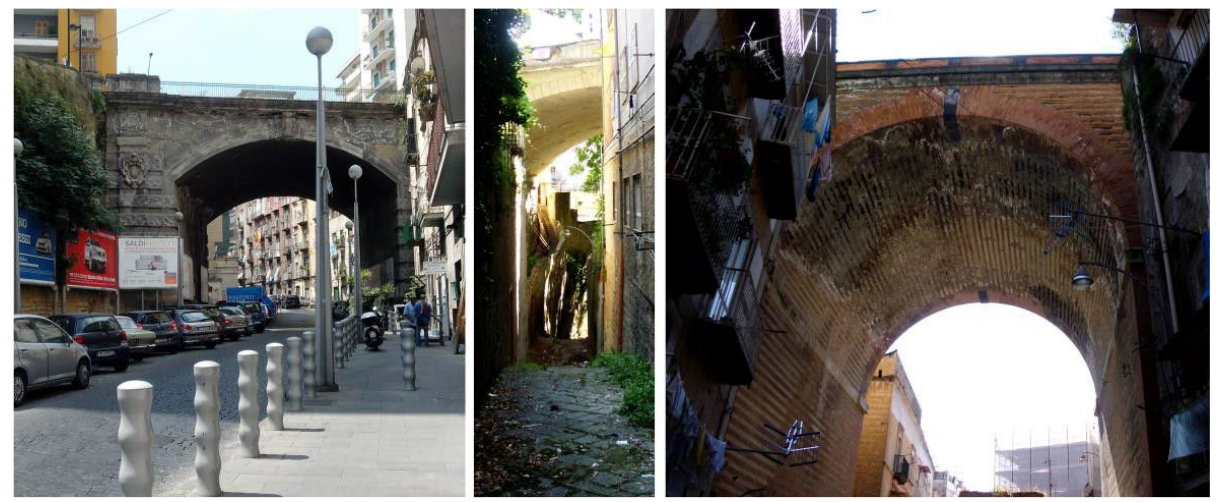

Fig. 13. Ponti/cavalcavia al Vomero. Ponte di via Suarez su via Conte della Cerra, ponte di via Girolamo Santacroce su vico Cacciottoli e ponte di Corso Vittorio Emanuele su vico Cacciottoli (Foto dell'autore).

Per realizzare il collegamento tra i due rioni Vomero e Arenella ${ }^{34}$. fu necessaria la realizzazione dei ponti di via Suarez e di via Mario Fiore (Fig. 13). Ancora un altro ponte/cavalcavia fu realizzato su via Annella di Massimo, l'antica via verso l'Arenella, affinché la nuova strada di via Scarlatti potesse

30 DE SETA C., a cura di, L'architettura a Napoli tra le due guerre, Napoli, Electa, 1999.

31 Gambardella A., De FAlCo C., Adolfo Avena architetto, Napoli, Electa, 1991.

32 Ricordiamo ad esempio la palazzina angolo via Scarlatti-via Morghen demolita per costruire un condominio multipiano.

33 Nel successivo piano di urbanizzazione del 1926 a cui seguirono due piani particolareggiati del $1937 \mathrm{e}$ del 1941, viene definita la sistemazione dei lotti e della rete viaria dell'Arenella.

34 ANONIMO, «Il nuovo rione all'Arenella», in Bollettino del Comune di Napoli, 1927, n. 2, pp. 9-14. 
proseguire verso Belvedere, nell'attuale via Francesco Cilea, per alcuni tratti sopraelevata rispetto all'originario livello stradale.

Solo più tardi, nelle zone più scoscese della collina del Vomero, sul versante meridionale, su un terreno degradante verso il mare, furono invece costruite strade con tornanti con visuali sul golfo. Furono costruite via Aniello Falcone che dalla Floridiana a mezza costa arrivava fino alla via Tasso, via Luigia Sanfelice e via Palizzi, tutte a carattere residenziale. Durante i lavori di ampliamento la Banca Tiberina ${ }^{35}$ si impegnò a rispettare, compatibilmente con le esigenze del piano, le preesistenti ville nobiliari, alcune delle quali sono ancora presenti nel tessuto edilizio attuale, altre, invece, andarono distrutte. È il caso, ad esempio, di villa Doria ${ }^{36}$ e di villa De Marinis ${ }^{37}$, che furono demolite per la risistemazione della zona avvenuta con la costruzione del ponte di via Francesco Cilea.

Nell'ambito della pianificazione urbana della città fu prevista, all'inizio del Novecento, la realizzazione di alcune aree da destinare a verde pubblico all'interno del nuovo quartiere, alcune delle quali probabilmente programmate anche per mediare l'innesto tra il preesistente tessuto urbano e l'impianto del nuovo quartiere.

Alcune di queste zone verdi, sia pure per brevi tratti, si sono conservate, come ad esempio i giardinetti di via Giambattista Ruoppolo, altre, invece, sono state edificate in seguito ad ulteriori varianti di piano. Questi punti nodali che possiamo definire di "cerniera" tra il vecchio e il nuovo, sono oggi ancora percepibili laddove un tempo si estendevano gli antichi Casali.

All'inizio del Novecento si completa la costruzione dei lotti, prevista nel piano di ampliamento del 1885, con edifici meno monumentali e più legati alle nuove correnti architettoniche. Pur rimanendo inalterato il sistema viario cambiano le cortine edilizie e la consistenza dell'isolato che, frammentato in piccole costruzioni, perde il carattere omogeneo della tipologia uniforme di una scacchiera, ormai svuotata dei suoi contenuti stilistici originari. Molte palazzine liberty furono costruite soprattutto in via Giuseppe Bonito, via Tito Angelini e via Domenico Cimarosa. É infatti in questa parte della collina, immediatamente in prossimità di Castel Sant'Elmo, che si è maggiormente conservata

35 Cf. nota 29.

36 La villa fu costruita nel 1865 circondata da un ampio giardino tra via Francesco Solimena, via Annella di Massimo e via Alessandro Scarlatti da Biagio Doria, dimora del nipote Gino, storiografo, Cf. LA GALA, A., Il Vomero e l'Arenella. Una storia per immagini, Napoli, Guida, 2002, p. 139.

37 La villa fu costruita su disegno di Antonio Costa tra via Scarlatti e via Mattia Preti. Cf. LA GALA, A., Vomero. Storia e storie, Napoli, Guida, 2004, p. 274. 
l'immagine dell'urbanizzazione di fine Ottocento-inizio Novecento. Il lato sud della collina del Vomero, da cui ancora oggi è possibile godere il paesaggio sul golfo, degrada verso la pianura seguendo una forte pendenza con un sistema di strade a tornanti, costituito principalmente da via Luigia Sanfelice, via Gioacchino Toma, via Filippo Palizzi, che termina con strade pedonali o con rampe e vie gradonate che si collegano alla sottostante strada del corso Vittorio Emanuele. Questo particolare sistema viario ha sottratto la zona al traffico e ad una edilizia più intensiva. Sfruttando il dislivello del terreno, fu possibile costruire anche edifici di quattro o cinque livelli senza ostacolare la vista verso il mare; in alcuni casi fu anche utilizzata una particolare tipologia con ingresso a ponte che collegava la parte alta dell'edificio alla strada. Ė il caso, ad esempio, della palazzina Russo Ermolli progettata da Stanislao Sorrentino e realizzata tra il 1915 e il 1918 con ricche decorazioni floreali (Fig. 14).

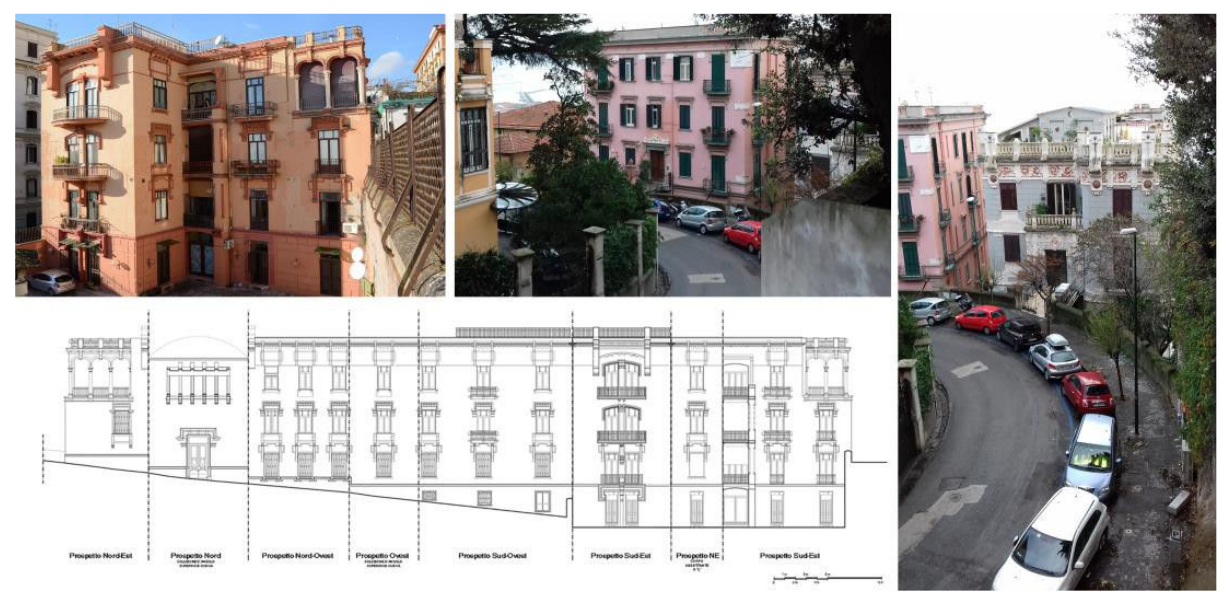

Fig. 14. Villa Loreley in via Toma, di cui si riporta il disegno delle facciate in un'unica composizione lineare (Martone, Giugliano, 2019); al centro: civico 52 di via Palizzi, a destra: palazzina Russo Ermolli.

Anche questa parte della collina conserva l'aspetto originario, austero ed elegante per la presenza ancora di palazzine e villini in stile Liberty del primo Novecento. Tra queste ville, in particolare villa Loreley di Adolfo Avena presenta particolari caratteri formali ed espressivi propri dell'architettura del floreale che sono stati studiati ed analizzati attraverso un rilievo digitale delle facciate eseguito con applicazioni di structure from motion, i cui esiti sono stati di recente pubblicati ${ }^{38}$ (Fig. 15).

38 L'esito della ricerca è stato pubblicato in: MARTONE, M., GIUGLIANO, A. M., «Le facciate architettoniche disegnate da Adolfo Avena. Il caso di palazzo Loreley», in Riflessioni Reflections l'arte del disegno/il 


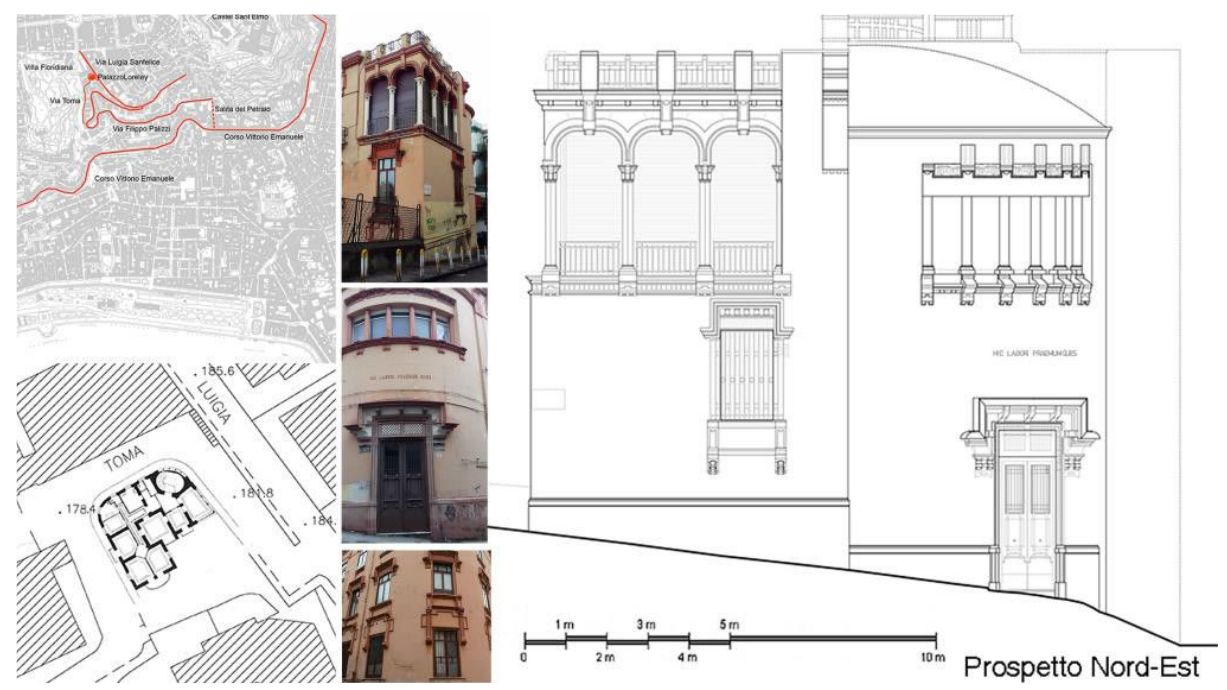

Fig. 15. Palazzina Loreley in via Toma di Adolfo Avena. Inquadramento cartografico, vedute fotografiche, restituzione dal rilievo digitale (Martone, Giugliano 2019).

La restituzione del rilievo con disegni a diverse scale ha permesso una lettura del manufatto sia nel suo contesto urbano di via Toma, sia a scala architettonica e di dettaglio, andando ad arricchire la conoscenza di un quartiere. Il rilievo ha consentito, infatti, una lettura unitaria delle facciate che si sono manifestate elementi caratterizzanti lo spazio urbano di uno dei luoghi più panoramici della collina del Vomero (Fig. 16).

Lo "spazio vissuto" del Vomero rappresentato nelle riviste illustrate

Il progetto di ampliamento della città di Napoli sulla collina del Vomero ebbe un forte risalto dalla stampa nazionale e straniera in quanto rappresentò un'importante questione architettonica e urbanistica con riscontri nel dibattito culturale europeo. Dalle pagine, infatti, dell'Illustrazione italiana, a cui si affiancarono riviste locali come il Bollettino del Comune di Napoli, il Bollettino del Collegio degli Ingegneri e Architetti di Napoli e il Poliorama pittoresco, si evince un ricco dibattito sui criteri progettuali della nuova edificazione ${ }^{39}$. Anche l'inviato straniero delle testate giornalistiche europee è colpito dalla città di Napoli per il suo panorama. All'interno delle riviste de The Illustrated London News e dell'Illustration di Parigi viene raffigurata la città con le famose vedute del golfo aventi sullo sfondo la verde collina del Vomero. L'arte

disegno dell'arte the art of drawing/the drawing of art, Roma, Gangemi, 2019, pp.793-800. Cf. MARTONE, M. 2020.

39 MALPICA, C., «Un viaggio sentimentale al Vomero», in Poliorama, 1836, anno I, II semestre, p. 394. 
dell'immagine visiva vive tra Ottocento e Novecento un forte impulso che trova espressione proprio nell'apparato iconografico delle riviste illustrate ${ }^{40}$.

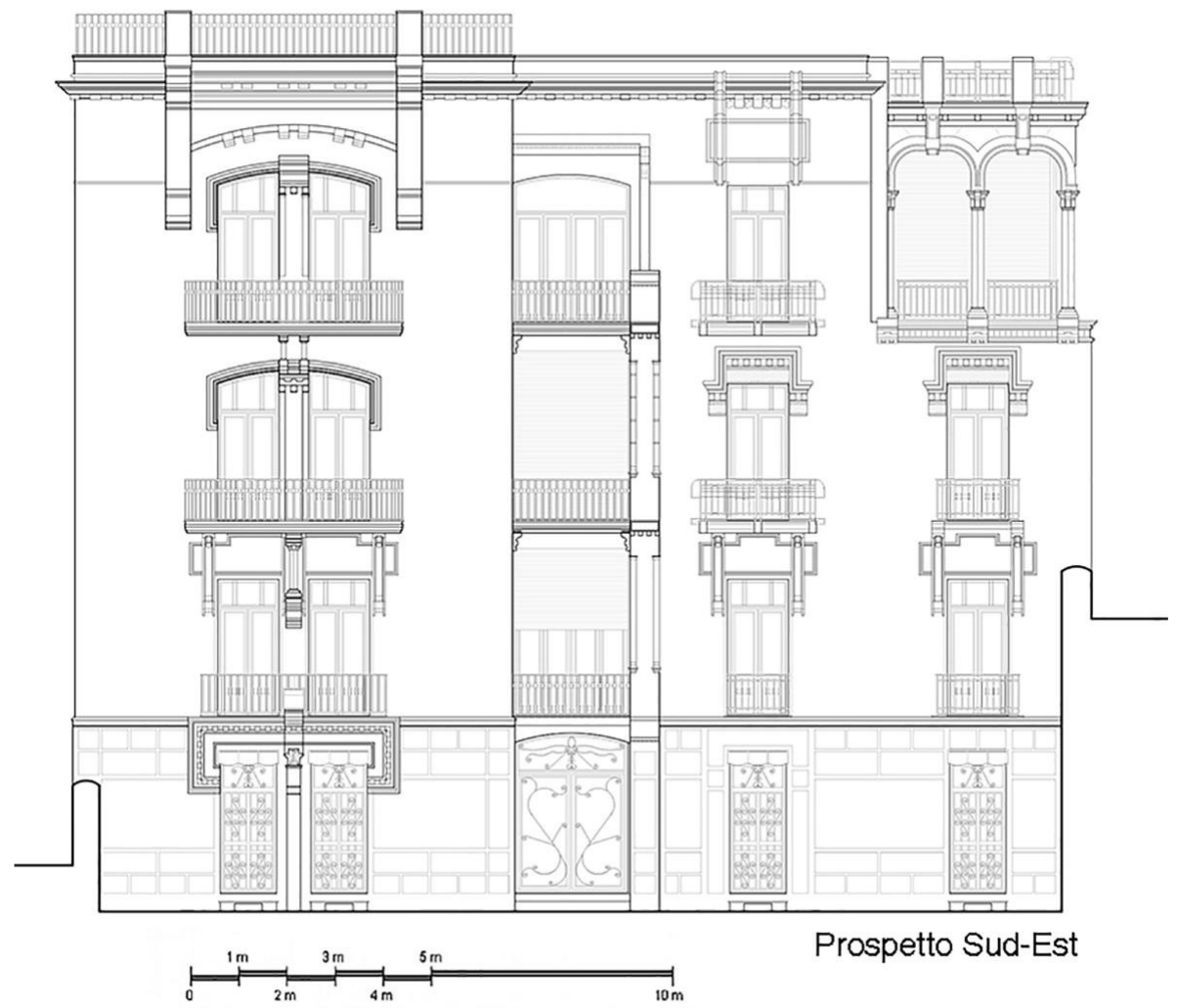

Fig. 16. Villa Loreley in via Toma di Adolfo Avena. Restituzione dal rilievo digitale (Martone, Giugliano, 2019).

Dalle rappresentazioni romantiche dei viaggiatori del Gran Tour del ' 700 legate alla cultura del pittoresco, si passa alla rappresentazione di paesaggi costruiti in cui vengono evidenziate le nuove architetture ed infrastrutture presenti sul territorio urbano ed extraurbano, come i ponti in ferro, le gallerie, $i$ trafori, le prime metropolitane, le architetture effimere delle Esposizioni nazionali e internazionali, con l'obiettivo di divulgare gli usi e i costumi di una società che si andava man mano a trasformare (Fig. 17). La lettura di alcune

40 MARTONE, M., «Graphic narration of places: the Vomero through the illustrated magazines between '800 and' 900», in MARCOS, C. L., editor, Graphic Imprints: The influence of rapresentation and ideation tools in architecture, Springer International Publishing AG, part of Springer Nature, Cham, Switzerland 2019, pp. 845-856. 
delle immagini di questo vasto apparato iconografico pubblicato sulle riviste nazionali e locali ha consentito di ritrovare rappresentazioni legate agli ambienti urbani del nuovo quartiere di ampliamento sulla collina e a scene di vita quotidiana che hanno contribuito a definire la storia dei luoghi testimoniando un periodo di intensa attività architettonica che ha modificato l'aspetto della collina del Vomero.

L'illustrazione, diversamente dalla rappresentazione pittorica di un quadro, esprimeva da un lato la volontà di fornire informazioni sulla vita sociale $\mathrm{e}$ culturale in un modo più immediato rispetto ad un documento scritto, e dall'altro lato anche la volontà di stupire con rappresentazioni comprensive di ogni dettaglio, talvolta riprodotte su intere pagine o su pagine doppie. Diverse sono le tecniche di rappresentazione: dalla xilografia su legno, all'acquaforte, alla litografia, tecniche che porteranno poi alla fotoxilografia e infine alla fotografia che troverà un'ampia applicazione nella documentazione dell'architettura e della città soprattutto dopo il primo conflitto mondiale.

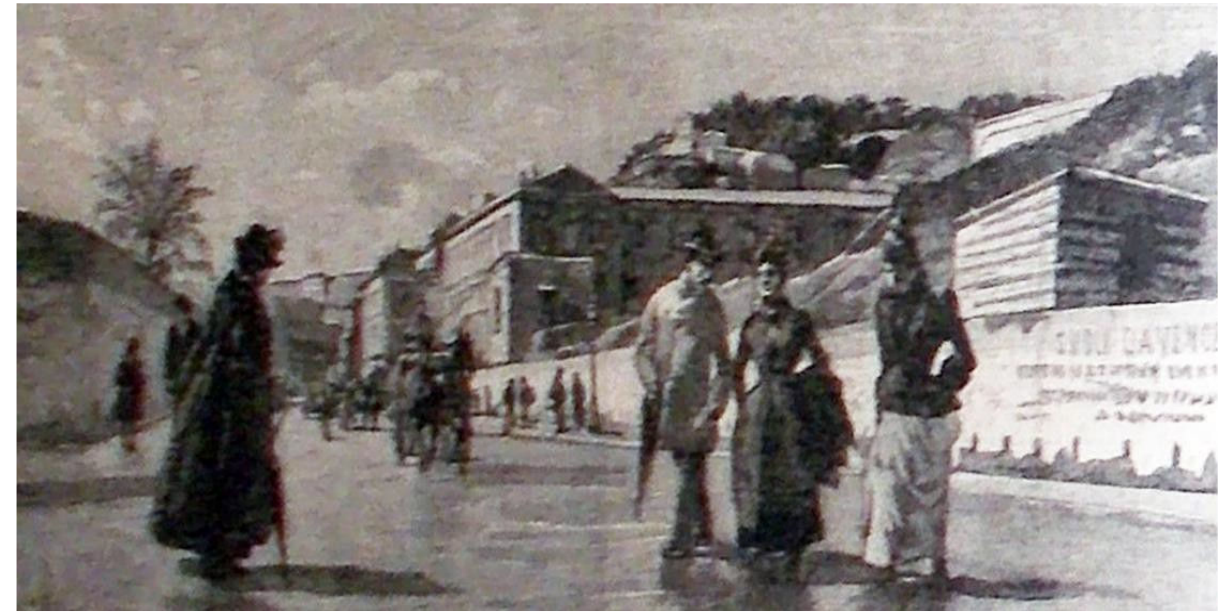

Fig. 17. La collina del Vomero vista da via dei Mille, L'Illustrazione italiana, n. $10,05 / 03 / 1893$, p. 161.

Nella rivista L'Illustrazione italiana del 7 giugno del 1891 in un articolo dal titolo "Nuova Napoli. Il Vomero", il giornalista Nicola Lazzaro, incredulo della bellezza del quartiere, costruito secondo le moderne esigenze abitative, fornito di acqua, con palazzi circondati da ampie strade, viali alberati e da raccolte piazze, scrive del Vomero come luogo dal quale con un solo sguardo si poteva percepire lo splendido panorama di Napoli. Collegato in pochi minuti e con pochissima spesa, con le funicolari il nuovo quartiere divenne, come 
afferma Lazzaro, il più elegante della città perché il più ameno e salubre. Nello stesso numero sono proposti i disegni che raffigurano le stazioni superiore e inferiore della nuova funicolare di Montesanto tra cui la stazione intermedia sul corso Vittorio Emanuele riportante la scritta Funicolare al Vomero ${ }^{41}$. Ricche rappresentazioni iconografiche, anche a tutta pagina con disegni dal vero o tratti da fotografie, ritraggono sulle pagine de L'Illustrazione italiana il centro del nuovo quartiere. Ricordiamo alcuni disegni di Gennaro Amato, pubblicati nel numero del 6 novembre 1892, raffiguranti paesaggi urbani del nuovo quartiere. Viene proposta una vista su via Tasso, una delle nuove strade costruite per collegare il Vomero con il resto della città. Nella stessa pagina Amato rappresenta una veduta su piazza Vanvitelli animata da persone che passeggiano, vivendo uno spazio urbano caratterizzato dalla presenza delle facciate neoclassiche degli edifici; sullo sfondo, con uno scorcio prospettico, è disegnato l'ingresso della stazione della funicolare di Chiaia. Accanto, il disegno di una lavannara (lavandaia), ci ricorda che questo era uno dei mestieri più diffusi, a quel tempo, sulla collina del Vomero ${ }^{42}$. Con l'apertura dell'acquedotto Serino anche verso la collina, le lavandaie si ritrovarono ad avere un lavoro sicuro, rafforzando così la loro presenza al Vomero. Purtroppo, non è rimasta alcuna traccia dei lavatoi utilizzati che un tempo caratterizzarono alcuni spazi soprattutto verso via Belvedere (Fig. 18).

Dalle pagine delle riviste illustrate partì anche una critica al nuovo quartiere soprattutto per quanto riguarda l'impatto ambientale che ne derivò. Molto perplesso nei confronti del nuovo progetto di ampliamento della città di Napoli sulla collina del Vomero e all'Arenella, fu il direttore del Bollettino del Collegio degli Ingegneri e Architetti, ing. Gennaro Pepe, che dalle pagine del suo giornale nel numero 2-3 del 1 febbraio 1886, mette in evidenza le inopportunità e il danno che una edificazione così intensa dei due rioni Vomero e Arenella potrà arrecare alla città, rischiando di perdere «quanto di più bello ha creato il sorriso della natura in quei luoghi»15. A tal scopo l'ingegnere Pepe suggerisce una edificazione ridotta e distribuita in modo tale da consentire da tutte le abitazioni previste di percepire liberamente il panorama e conservare la vista dei campi. L'inviato da Napoli, Gennaro Amato dell'Illustrazione Italiana, evidenziò gli interessi che spinsero gli imprenditori ad investire nell'edilizia al Vomero favorendo scelte architettoniche in contrasto con la fantasia della città di Napoli.

41 LAZZARO, N., «Napoli nuova. Il Vomero», in L'Illustrazione italiana, anno XVIII, n. 23, 7 giugno 1891, pp. 354-356.

42 AMATO, G., 1892, «Le nostre incisioni. Vomero-Rione nuovo», in L'Illustrazione italiana, 6 novembre 1892, n. 45, pp. 308 e 311; L'Illustrazione italiana, 07/06/1891, n. 23 p. 356. 

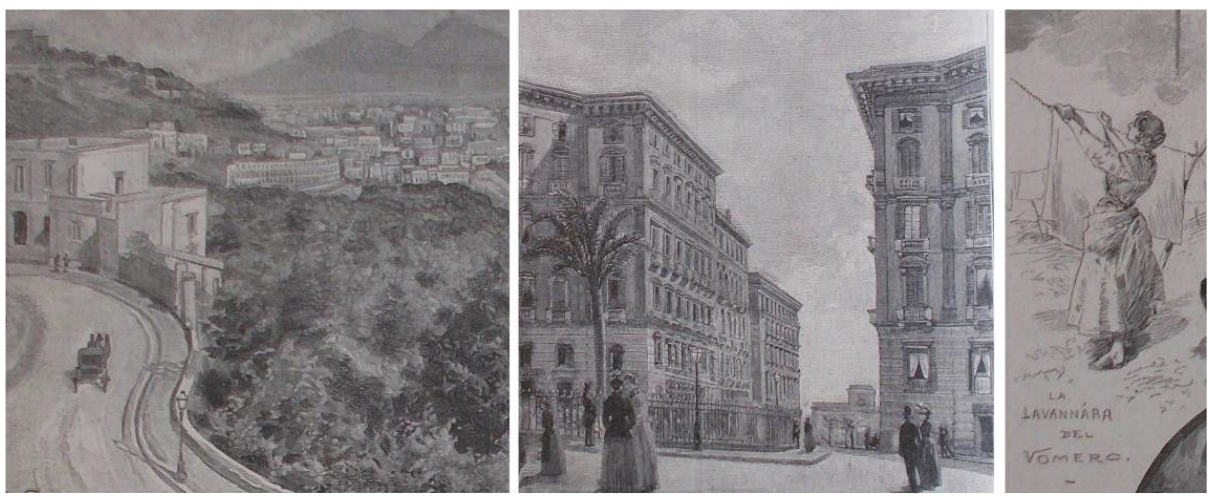

Fig. 18. Disegni raffiguranti: via Tasso, piazza Vanvitelli, la "lavannara" del Vomero, da: L'Illustrazione italiana, 06/11/1892, p. 308.

$\mathrm{Fu}$ riscontrata una forte ripetitività nella tipologia edilizia scelta per la costruzione del nuovo quartiere, con palazzi che in nulla si differenziavano rispetto a quelli costruiti a Torino o a Roma ${ }^{43}$. Dal confronto con foto attuali che ritraggono il Vomero nel suo stato di fatto, eseguite cercando di rispettare quasi lo stesso punto di vista delle vedute pubblicate sulle riviste, è stato possibile riconoscere gli edifici ed elementi architettonici dell'antico impianto urbano del quartiere del Vomero, che rappresentano i segni tangibili di un patrimonio culturale su cui prendono forma i paesaggi culturali percepiti da chi percorre il quartiere. Il Vomero, quindi, con l'urbanizzazione di fine Ottocento diventa uno spazio di cui se ne appropria l'istituzione, ossia il governo della città, diventando un prodotto sociale ${ }^{44}$. Sul piano spaziale, è noto che "appropriazione" significa anche "delimitazione", infatti mentre prima come luogo di delizie il Vomero non aveva dei confini ben precisi, diventando un quartiere, ossia un luogo urbano, uno spazio sociale, assume l'identità di territorio con dei confini precisi. Il linguaggio, attraverso cui si realizza la forma urbana, è il linguaggio architettonico delle facciate, ora neoclassico ora liberty che caratterizza l'immagine visiva del nuovo quartiere.

Il Vomero a fine Ottocento si trasforma, quindi, in uno spazio progettato e pianificato da una ideologia dominante, che viene rappresentato nei disegni dei piani regolatori e di ampliamento. Lo spazio pensato realizzandosi interagisce

$43 \mathrm{Su}$ questo concetto furono redatti molti piani urbanistici in Europa e in Italia. Ricordiamo oltre a quello già citato di Hasmann per Parigi realizzato tra il 1852-1870 da Napoleone III, quello di Cerdà per Barcellona del 1859, il Ring di Vienna 1859-1872, di Lindhagen per Stoccolma del 1866, il piano dell'Antonelli del 1852 per la città di Torino, il piano Trotti per la città di Bari del 1867, il piano di Roma del 1883 e il piano del Poggi per la città di Firenze del 1885.

44 LEFEBVRE, H., op. cit. 
con l'ambiente naturale e con le persone che vi vanno ad abitare, trasformandosi ulteriormente in uno spazio vissuto che comprende uno spazio fisico, percepito e sociale, diventando uno spazio eterogeneo.

\section{CONCLUSIONI}

Il Vomero, come ogni luogo, è unico perché le ragioni per cui esiste non sono generalizzabili ad altri luoghi. Ogni luogo ha una storia propria, a fondamento della sua identità culturale riconoscibile. La dimensione locale è, infatti, un punto di vista che evidenzia peculiarità, identità, unicità di un luogo. Il termine "luogo" non fa riferimento a dimensioni spaziali, né fa ferimento ad una specifica scala grafica di rappresentazione. Sono luoghi: un paese, una città, una valle, una piazza, un centro storico, un quartiere, se descritti, analizzati, interpretati e trattati nei loro caratteri identitari peculiari ${ }^{45}$. Si ritiene questo il punto di partenza per sottolineare l'importanza della tutela e della valorizzazione dei paesaggi culturali.

L'immagine del Vomero così come viene percepita oggi ci restituisce, anche se a fatica, brani di storia che, sulla base di una documentazione critica dei luoghi, ancora si riconoscono come frammenti sopravvissuti che vanno tutelati e conservati per il valore fondamentale di testimonianza che essi esprimono, di una storia che va conosciuta per essere conservata. Si ritiene, pertanto, che la rappresentazione, nei suoi molteplici aspetti, ricopra un ruolo fondamentale nella documentazione iconografica di un luogo e possa, quindi, contribuire a divulgare la conoscenza come primo passo verso la tutela e la salvaguardia di ciò che è rimasto del Vomero antico come bene comune territoriale.

Purtroppo, bisogna constatare che nel tessuto urbano attuale del Vomero le immagini dell'originario luogo di delizie e della città ottocentesca risultano o cancellate $\mathrm{o}$ fortemente alterate dall'edilizia speculativa della città contemporanea e da numerosi interventi pubblici e privati. Elementi costruttivi, di ristrutturazione e di arredo urbano indifferenti ai luoghi sono stati introdotti nell'ambiente distruggendone l'identità locale, agendo da detrattori ambientali e paesaggistici. Tuttavia, è in questo spazio ibrido, eterogeneo, in cui si legge una stratificazione "disordinata" di interventi urbanistici ed architettonici, che

45 Cf. http://www.societadeiterritorialisti.it/wp-content/uploads/2013/05/110221_manifesto.societ.terri torialista.pdf (giugno 2021). 
si manifestano ancora paesaggi culturali fragili che vanno difesi e tutelati da un continuo processo di degrado.

Per effetto, anche, della globalizzazione, l'uso attuale che viene fatto del territorio favorisce una pericolosa decontestualizzazione dei luoghi, dei paesaggi, degli ambienti di vita delle popolazioni e delle relazioni sociali, in quanto mira principalmente ad aumentare il benessere individuale e sociale di coloro che abitano, lavorano o percorrono un determinato luogo. Per contrapporsi a questa logica è necessario aumentare la conoscenza, la divulgazione e la comunicazione dei valori culturali presenti nel Vomero attraverso campagne di rilievo e di documentazione critica sia a livello urbano che architettonico nella consapevolezza che l'identità locale è innanzi tutto una potenzialità, un valore aggiunto, una risorsa per uno sviluppo sostenibile che ci consente di progettare il nostro prossimo futuro. Pertanto, è nella natura dinamica dei paesaggi culturali che si può ancora leggere il Vomero in tutto il suo essere luogo, spazio e territorio.

\section{BIBLIOGRAFIA}

ALISIO, G., Il Vomero, Napoli, Electa, 1987. ALISIO, Il Vomero, Electa, Napoli 1987. CELANO, C., Notizie del bello, dell'antico e del curioso della città di Napoli, Napoli, 1692, nella Edizione a cura di CHIARINI, G. B., Napoli, Stamperia di Nicola Mencia, 1859, vol. IV, p. 745-753. Giornata Sesta.

COCCHIA, C., L'edilizia a Napoli tra il 1918 e il 1958, Napoli, Società pel Risanamento di Napoli, 1961.

DE SETA C., a cura di, L'architettura a Napoli tra le due guerre, Napoli, Electa, 1999. FOUCAULT, M., Spazi altri. I luoghi delle eterotopie, a cura di VACCARO S., Udine, Mimesis Edizioni, 2011.

GAMBARDELLA A., DE FALCO C., Adolfo Avena architetto, Napoli, Electa, 1991. LEFEBVRE, H., La produzione dello spazio, Milano, Pgreco Edizioni, 2018. SOJA, E., Thirdspace: Journeys to Los Angeles and Other Real and Imagined Places. Cambridge (USA), Blackwell, 1996.

MAGNAGHI, A., Il principio territoriale, Torino, Bollati Boringhieri, 2020.

MARTONE, M., «I paesaggi pontini: dalla macroscala all'elemento di dettaglio. Alcuni esempi», in CUNDARI, C., GIGLI, F. (a cura di). Paesaggi di città e territori. Roma, Aracne, 2011, p.100-116.

MARTONE, M., GIUGLIANO, A. M., «Le facciate architettoniche disegnate da Adolfo Avena. Il caso di palazzo Loreley», in Riflessioni Reflections l'arte 
del disegno/il disegno dell'arte the art of drawing/the drawing of art, Roma, Gangemi, 2019.

MARTONE, M., La collina del Vomero. Da paesaggio agreste a forma urbana, Roma, Aracne Editrice, 2020.

PALLOTTINO, P., Storia dell 'illustrazione italiana, Bologna, Zanichelli, 1988. PANE, R., VALERIO V., a cura di, La città di Napoli tra vedutismo e cartografia. Piante e vedute dal XV al XIX secolo., Grimaldi \& e C. Editori, Napoli 1987. PURINI F., «Un paese senza paesaggio», in Il disegno del paesaggio italiano, Casabella, n. 575-576, 1993.

RUSSO, G., Il Risanamento e l'Ampliamento della città di Napoli, Napoli, Società pel Risanamento, 1959.

SUMMONTE, A., Historia della città e Regno di Napoli, Napoli, Stamperia di Domenico Vivenzio, 1748, Tomo primo dedicato all'eccellentissima città di Napoli, p. 305-318.

WERNER J., Contributi alla Campania antica. La via Puteolis Neapolim, Napoli, Arte Tipografica, 1953.

\section{Maria Martone}

Dipartimento de Ingenieria Civile, Edile e Ambientale Università Sapienza di Roma https://orcid.org/0000-0002-8771-5628 maria.martone@uniroma1.it 